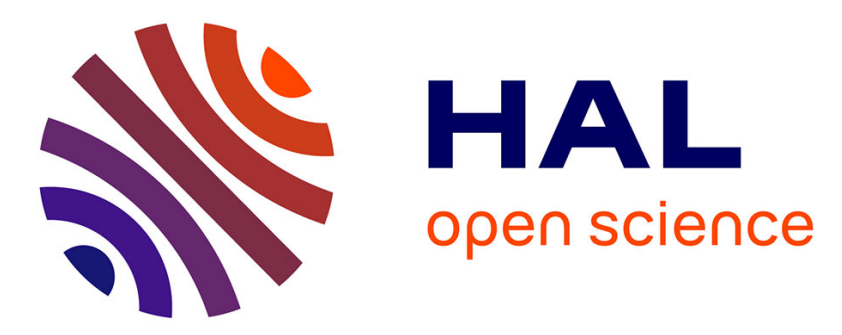

\title{
Indirect controllability of locally coupled systems under geometric conditions
}

Fatiha Alabau-Boussouira, Matthieu Léautaud

\section{To cite this version:}

Fatiha Alabau-Boussouira, Matthieu Léautaud. Indirect controllability of locally coupled systems under geometric conditions. Comptes Rendus. Mathématique, 2011, 349 (7-8), pp.395-400. 10.1016/j.crma.2011.02.004 . hal-00617910

\section{HAL Id: hal-00617910 https://hal.science/hal-00617910}

Submitted on 30 Aug 2011

HAL is a multi-disciplinary open access archive for the deposit and dissemination of scientific research documents, whether they are published or not. The documents may come from teaching and research institutions in France or abroad, or from public or private research centers.
L'archive ouverte pluridisciplinaire HAL, est destinée au dépôt et à la diffusion de documents scientifiques de niveau recherche, publiés ou non, émanant des établissements d'enseignement et de recherche français ou étrangers, des laboratoires publics ou privés. 


\title{
Indirect controllability of locally coupled systems under geometric conditions
}

\author{
Fatiha Alabau-Boussouira*, Matthieu Léautaud ${ }^{\dagger *}$
}

December 8, 2010

\begin{abstract}
We consider systems of two wave/heat/Schrödinger-type equations coupled by a zero order term, only one of them being controlled. We prove an internal and a boundary null-controllability result in any space dimension, provided that both the coupling and the control regions satisfy the Geometric Control Condition. This includes several examples in which these two regions have an empty intersection.
\end{abstract}

\begin{abstract}
Contrôlabilité indirecte de systèmes localement couplés sous des conditions géométriques. On s'intéresse à des systèmes constitués de deux équations d'ondes, de la chaleur ou de Schrödinger, couplées par un terme d'ordre zéro, et dont seulement l'une est controlée. En supposant que les zones de couplage et de contrôle satisfont toutes deux la Condition Géométrique de Contrôle, on montre un résultat de contrôle interne et frontière en dimension quelconque d'espace. Ceci fournit de nombreux exemples pour lesquels ces deux régions ne s'intersectent pas.
\end{abstract}

\section{Version française abrégée}

Durant les dix dernières années, les propriétés de contrôlabilité des systèmes paraboliques du type

$$
\begin{cases}e^{i \theta} u_{1}^{\prime}-\Delta_{c} u_{1}+a u_{1}+\delta p u_{2}=b f & \text { dans }(0, T) \times \Omega, \\ e^{i \theta} u_{2}^{\prime}-\Delta_{c} u_{2}+a u_{2}+p u_{1}=0 & \text { dans }(0, T) \times \Omega, \\ u_{1}=u_{2}=0 & \text { sur }(0, T) \times \partial \Omega, \\ \left.\left(u_{1}, u_{2}\right)\right|_{t=0}=\left(u_{1}^{0}, u_{2}^{0}\right) & \text { dans } \Omega,\end{cases}
$$

avec $\theta=0$, ont été étudiées intensivement. Ici, $a, b, c, p$ sont des fonctions réelles régulières de $x \in \Omega$, avec $b \geq 0$ et $p \geq 0$, $\delta>0$ est un paramètre, $-\Delta_{c}$ est un opérateur autoadjoint uniformément elliptique sur $\Omega$, et $f$ est le contrôle. Le résultat général concernant ces systèmes, prouvé par différentes méthodes dans $[16,4,7,9]$ est un théorème de contrôlabilité à zéro dès qu'on suppose $\{p>0\} \cap\{b>0\} \neq \emptyset$. Qu'en est-il du cas $\{p>0\} \cap\{b>0\}=\emptyset$ ? Le second problème auquel on s'intéresse ici est le problème du contrôle frontière associé à (1) (cf.(4)). S'il semble résolu en dimension 1 d'espace pour $a$ et $p$ constants (voir [6]), il reste complètement ouvert en dimension supérieure ou pour des coefficients variables. Pour ces deux problèmes, il semblerait que la théorie des équations paraboliques et les outils utilisés se heurtent pour le moment à de sérieuses difficultés. D'autre part, on sait depuis [15] que les propriétés de contrôlabilité des équations hyperboliques se transmettent aux équations paraboliques. Poursuivant l'étude initiée dans [2], nous répondons aux deux questions ci-dessus pour le système hyperbolique (3) (consistant à remplacer $e^{i \theta} u_{j}^{\prime}$ par $u_{j}^{\prime \prime}$, pour $j=1,2$, dans (1)) sous certaines hypothèses. Nous en déduisons ensuite une réponse partielle à ces questions pour (1).

Dans le cadre du contrôle frontière, on renvoie aux systèmes (4) et (5) ci-dessous, pour lesquelles le contrôle agit par la condition au bord $\left.u_{1}\right|_{\partial \Omega}=b_{\partial} f$, avec $b_{\partial} \in \mathscr{C}_{c}^{\infty}(\partial \Omega), b_{\partial} \geq 0$. On appellera GCC (resp. GCC $C_{\partial}$ ) la condition de contrôle géométrique interne (resp. frontière) de [5], que nous rappelons dans la section 2.

Pour formuler nos résultats, on utilisera les hypothèses suivantes:

(i) L'Opérateur $-\Delta_{c}+a$ est uniformément coercif sur $\Omega$.

(ii) On a $\{p>0\} \supset \bar{\omega}_{p}$ pour un ouvert $\omega_{p} \subset \Omega$ et on pose $p^{+}:=\|p\|_{L^{\infty}(\Omega)}$.

(iii) On a $\{b>0\} \supset \bar{\omega}_{b}$ (resp. $\left\{b_{\partial}>0\right\} \supset \bar{\Gamma}_{b}$ ) pour un ouvert $\omega_{b} \subset \Omega$ (resp. $\Gamma_{b} \subset \partial \Omega$ ).

\footnotetext{
*LMAM, Université Paul Verlaine-Metz and CNRS (UMR 7122), 57045 Metz Cedex 1, France. e-mail: alabau@univ-metz . fr

†Université Pierre et Marie Curie Paris 6, UMR 7598, Laboratoire Jacques-Louis Lions, Paris, F-75005 France; CNRS, UMR 7598 LJLL, Paris, F-75005 France. e-mail: leautaud@ann.jussieu.fr

${ }^{\ddagger}$ Laboratoire POEMS, INRIA Paris-Rocquencourt/ENSTA, CNRS UMR 2706, France.
} 
Theorem 0.1 (Systèmes d'équations d'ondes). On suppose que (i) est satisfaite, que $\omega_{p}$ satisfait GCC, et que $\omega_{b}$ (resp. $\left.\Gamma_{b}\right)$ satisfait GCC (resp. GCC $C_{\partial}$ ). Il existe alors une constante $\delta_{*}>0$ telle que pour tout $\left(\delta, p^{+}\right)$satisfaisant $\sqrt{\delta} p^{+}<\delta_{*}$, il existe un temps $T_{*}>0$ tel que pour tout $T>T_{*}$, tous $p, b$ (resp. $b_{\partial}$ ) satisfaisant (ii) et (iii), et toutes données initiales $\left(u_{1}^{0}, u_{2}^{0}, u_{1}^{1}, u_{2}^{1}\right) \in H_{0}^{1}(\Omega) \times H^{2} \cap H_{0}^{1}(\Omega) \times L^{2}(\Omega) \times H_{0}^{1}(\Omega)\left(\right.$ resp. $\left.\left(u_{1}^{0}, u_{2}^{0}, u_{1}^{1}, u_{2}^{1}\right) \in L^{2}(\Omega) \times H_{0}^{1}(\Omega) \times H^{-1}(\Omega) \times L^{2}(\Omega)\right)$, il existe un contrôle $f \in L^{2}((0, T) \times \Omega)$ (resp. $f \in L^{2}((0, T) \times \partial \Omega)$ ) tel que la solution de (3) (resp. (5)) vérifie $\left.\left(u_{1}, u_{2}, u_{1}^{\prime}, u_{2}^{\prime}\right)\right|_{t=T}=0$.

On remarque que les espaces dans lesquels $u_{1}$ et $u_{2}$ sont contrôlés ne sont pas les mêmes, ce qui est naturel. Ce résultat est montré dans un cadre abstrait (voir Section 3), incluant aussi des systèmes de plaques couplées. On en déduit, grâce aux méthodes de transmutation de $[15,11,13,12]$ les résultats suivants pour deux équations de diffusion ou de Schrödinger couplées.

Theorem 0.2 (Systèmes d'équations de diffusion). On suppose que (i) est satisfaite, que $\omega_{p}$ satisfait GCC, et que $\omega_{b}$ (resp. $\Gamma_{b}$ ) satisfait GCC (resp. GCC $C_{\partial}$ ). Il existe alors une constante $\delta_{*}>0$ telle que pour tout $\left(\delta, p^{+}\right)$satisfaisant $\sqrt{\delta} p^{+}<\delta_{*}$, pour tout $T>0, \theta \in(-\pi / 2, \pi / 2)$, pour tous $p, b$ (resp. $b_{\partial}$ ) satisfaisant (ii) et (iii), et toutes données initiales $\left(u_{1}^{0}, u_{2}^{0}\right) \in\left(L^{2}(\Omega)\right)^{2}$ (resp. $\left.\left(u_{1}^{0}, u_{2}^{0}\right) \in\left(H^{-1}(\Omega)\right)^{2}\right)$, il existe un contrôle $f \in L^{2}((0, T) \times \Omega)\left(\right.$ resp. $f \in L^{2}((0, T) \times \partial \Omega)$ ) tel que la solution de (1) (resp. (4)) vrifie $\left.\left(u_{1}, u_{2}\right)\right|_{t=T}=0$.

Ce résultat répond donc partiellement aux deux questions posées, fournissant de nombreux exemples pour lesquels $\{p>$ $0\} \cap\{b>0\}=\emptyset$ ainsi qu'un résultat de contrôle frontière en toute dimension d'espace pour un couplage variable. Cependant, on notera que les hypothèses géométriques ne sont pas naturelles pour des équations paraboliques. Par conséquent, le théoreme 0.2 n'est qu'un premier pas pour cette étude.

Theorem 0.3 (Systèmes d'équations de Schrödinger). Sous les hypothèse du théorème 0.2, le même résultat de contrôlabilité est valide pour le système (1) (resp. (4)) avec $\theta= \pm \pi / 2$, pour des données initiales $\left(u_{1}^{0}, u_{2}^{0}\right) \in L^{2}(\Omega) \times H_{0}^{1}(\Omega)$ (resp. $\left.\left(u_{1}^{0}, u_{2}^{0}\right) \in H^{-1}(\Omega) \times L^{2}(\Omega)\right)$.

\section{Introduction}

During the last decade, the controllability properties of coupled parabolic equations like

$$
\begin{cases}e^{i \theta} u_{1}^{\prime}-\Delta_{c} u_{1}+a u_{1}+\delta p u_{2}=b f & \text { in }(0, T) \times \Omega, \\ e^{i \theta} u_{2}^{\prime}-\Delta_{c} u_{2}+a u_{2}+p u_{1}=0 & \text { in }(0, T) \times \Omega, \\ u_{1}=u_{2}=0 & \text { on }(0, T) \times \partial \Omega, \\ \left.\left(u_{1}, u_{2}\right)\right|_{t=0}=\left(u_{1}^{0}, u_{2}^{0}\right) & \text { in } \Omega,\end{cases}
$$

with $\theta=0$, have been intensively studied. Here, $\Delta_{c}$ is a selfadjoint elliptic operator, and all the parameters are precisely defined in Section 2. The null-controllability problem under view is the following: given a time $T>0$ and initial data, is it possible to find a control function $f$ so that the state has been driven to zero in time $T$ ? It has been proved in $[16,4,7,9]$ with different methods that System (2) is null-controllable as soon as $\{p>0\} \cap\{b>0\} \neq \emptyset$. In these works, the case $\{p>0\} \cap\{b>0\}=\emptyset$ has been left as an open problem. However, Kavian and de Teresa [8] have proved for a cascade system (i.e. taking $\delta=0$ in (2)) that approximate controllability holds. The natural question is then whether or not nullcontrollability (which is a stronger property) still holds in the case $\{p>0\} \cap\{b>0\}=\emptyset$ ?

The second Problem under interest here is the boundary controllability of systems like (2) (or more precisely System (4) below). The recent work [6] studies slightly more general systems in one space dimension and with constant coupling coefficients. The cases of higher space dimensions and varying coupling coefficients (and in particular when the coefficients vanish in a neighborhood of the boundary) are to our knowledge completely open.

Concerning these two open problems, it seems that the parabolic theory and associated tools encounter for the moment some essential difficulties.

On the other hand, it is known from [15] that controllability properties can be transferred from hyperbolic equations to parabolic ones. And it seems, at least for boundary control problems, that the theory for coupled hyperbolic equations of the type

$$
\begin{cases}u_{1}^{\prime \prime}-\Delta_{c} u_{1}+a u_{1}+\delta p u_{2}=b f & \text { in }(0, T) \times \Omega, \\ u_{2}^{\prime \prime}-\Delta_{c} u_{2}+a u_{2}+p u_{1}=0 & \text { in }(0, T) \times \Omega, \\ u_{1}=u_{2}=0 & \text { on }(0, T) \times \partial \Omega, \\ \left.\left(u_{1}, u_{2}, u_{1}^{\prime}, u_{2}^{\prime}\right)\right|_{t=0}=\left(u_{1}^{0}, u_{2}^{0}, u_{1}^{1}, u_{2}^{1}\right) & \text { in } \Omega,\end{cases}
$$

is better understood (see [2]), even less studied. In the case of varying coefficients and several space-dimensions, the associated stabilization problem is addressed in $[1,3]$. 
In the present work, we answer these two questions for hyperbolic problems improving the results of $[2,3]$, and then deduce a (partial) solution to the two open questions raised above. Indeed, we prove that Systems (2)-(3) are null-controllable (in appropriate spaces) as soon as $\{p>0\}$ and $\{b>0\}$ both satisfy the Geometric Control Condition (recalled below) and $\sqrt{\delta}\|p\|_{L^{\infty}(\Omega)}$ satisfies a smallness assumption. This contains several examples with $\{p>0\} \cap\{b>0\}=\emptyset$ in any space-dimension, and partially answers to the first question. We prove as well that the same controllability result holds for boundary control, which partially answers to the second question. Of course, the geometric conditions needed here are essential (and even sharp) for coupled waves, but inappropriate for parabolic equation. However, this is a first step towards a better understanding of these types of systems. In one space dimension in particular, the geometric conditions are reduced to a non-emptiness condition and are hence optimal for parabolic systems as well. Similar results have been obtained simultaneously in [14] with different methods in one space dimension for cascade systems.

In this note, we first state our results for wave/heat/Shrödinger-type Systems. Then, we introduced an abstract setting adapted to these problems and give some elements of the proof in this context.

Acknowledgements. The authors want to thank B. Dehman for discussions on the article [5], S. Ervedoza for having pointed out the papers [11, 12], and L. Miller for discussions on these two articles. The first author would like to thank the Fondation des Sciences Mathématiques de Paris and the organizers of the IHP trimester on control of PDE's for their support. The second author wishes to thank O. Glass and J. Le Rousseau for very fruitful discussions and encouragements. Both authors were partially supported by l'Agence Nationale de la Recherche under grant ANR-07-JCJC-0139-01 and the GDRE CONEDP (CNRS/INDAM/UP).

\section{Main results}

Let $\Omega$ be a bounded domain in $\mathbb{R}^{n}$ with smooth (say $\mathscr{C}^{\infty}$ ) boundary (or a smooth connected compact riemannian manifold with or without boundary) and $\Delta_{c}=\operatorname{div}(c \nabla)$ a (negative) Laplace operator (or Laplace Beltrami operator with respect to the riemannian metric) on $\Omega$. Here, $c$ denotes a smooth (say $\mathscr{C}^{\infty}$ ) positive symmetric matrix i.e. in particular $C_{0}^{-1}|\xi|^{2} \leq$ $c(x) \xi \cdot \xi \leq C_{0}|\xi|^{2}$ for some $C_{0}>1$, for all $x \in \bar{\Omega}, \xi \in \mathbb{R}^{n}$. We consider the control problems (2) with $\theta \in[-\pi / 2, \pi / 2]$, including Schrödinger-type systems for $\theta= \pm \pi / 2$ and diffusion-type systems for $\theta \in(-\pi / 2, \pi / 2)$, and (3) consisting in a wave-type system, with only one control force. In these systems, $a=a(x), p=p(x)$ and $b=b(x)$ are smooth real-valued functions on $\Omega, \delta>0$ is a constant parameter and $f$ is the control function, that can act on the system.

We shall also consider the same systems controlled from the boundary through the (smooth) real-valued function $b_{\partial}$ :

$$
\begin{gathered}
\begin{cases}e^{i \theta} u_{1}^{\prime}-\Delta_{c} u_{1}+a u_{1}+\delta p u_{2}=0 & \text { in }(0, T) \times \Omega, \\
e^{i \theta} u_{2}^{\prime}-\Delta_{c} u_{2}+a u_{2}+p u_{1}=0 & \text { in }(0, T) \times \Omega, \\
u_{1}=b_{\partial} f, u_{2}=0 & \text { on }(0, T) \times \partial \Omega, \\
\left.\left(u_{1}, u_{2}\right)\right|_{t=0}=\left(u_{1}^{0}, u_{2}^{0}\right) & \text { in } \Omega,\end{cases} \\
\begin{cases}u_{1}^{\prime \prime}-\Delta_{c} u_{1}+a u_{1}+\delta p u_{2}=0 & \text { in }(0, T) \times \Omega, \\
u_{2}^{\prime \prime}-\Delta_{c} u_{2}+a u_{2}+p u_{1}=0 & \text { in }(0, T) \times \Omega, \\
u_{1}=b_{\partial} f, u_{2}=0 & \text { on }(0, T) \times \partial \Omega, \\
\left.\left(u_{1}, u_{2}, u_{1}^{\prime}, u_{2}^{\prime}\right)\right|_{t=0}=\left(u_{1}^{0}, u_{2}^{0}, u_{1}^{1}, u_{2}^{1}\right) & \text { in } \Omega .\end{cases}
\end{gathered}
$$

We first notice that, on the space $\left(L^{2}(\Omega)\right)^{2}$ endowed with the inner product $(u, v)_{\delta}=\left(u_{1}, v_{1}\right)_{L^{2}(\Omega)}+\delta\left(u_{2}, v_{2}\right)_{L^{2}(\Omega)}$, the operator $A_{\delta}=\left(\begin{array}{cc}-\Delta_{c}+a & \delta p \\ p & -\Delta_{c}+a\end{array}\right)$, with domain $\mathcal{D}\left(A_{\delta}\right)=\left(H^{2}(\Omega) \cap H_{0}^{1}(\Omega)\right)^{2}$, is selfadjoint. As a consequence, for $f \in L^{2}((0, T) \times \Omega)$, the Cauchy problem (2), resp. (3), is well-posed in $\left(L^{2}(\Omega)\right)^{2}$, resp. $\left(H_{0}^{1}(\Omega)\right)^{2} \times\left(L^{2}(\Omega)\right)^{2}$, in the sense of semigroup theory. Then, taking $f \in L^{2}((0, T) \times \partial \Omega)$ the initial-boundary value problem (4), resp. (5), is well-posed in $\left(H^{-1}(\Omega)\right)^{2}$, resp. $\left(L^{2}(\Omega)\right)^{2} \times\left(H^{-1}(\Omega)\right)^{2}$, in the sense of transposition solution (see [10]).

An important remark to make before addressing the controllability problem is concerned with the regularity of solutions of (3)-(5). If one takes for system (3) (resp. (5)) an initial data $\left(u_{1}^{0}, u_{2}^{0}, u_{1}^{1}, u_{2}^{1}\right) \in H_{0}^{1}(\Omega) \times H^{2} \cap H_{0}^{1}(\Omega) \times L^{2}(\Omega) \times H_{0}^{1}(\Omega)$ (resp. $\left.\left(u_{1}^{0}, u_{2}^{0}, u_{1}^{1}, u_{2}^{1}\right) \in L^{2}(\Omega) \times H_{0}^{1}(\Omega) \times H^{-1}(\Omega) \times L^{2}(\Omega)\right)$, and a control $f \in L^{2}((0, T) \times \Omega)\left(\right.$ resp. $f \in L^{2}((0, T) \times \partial \Omega)$, then the state $\left(u_{1}, u_{2}, u_{1}^{\prime}, u_{2}^{\prime}\right)$ remains in the space $H_{0}^{1}(\Omega) \times H^{2} \cap H_{0}^{1}(\Omega) \times L^{2}(\Omega) \times H_{0}^{1}(\Omega)\left(\right.$ resp. $\left.L^{2}(\Omega) \times H_{0}^{1}(\Omega) \times H^{-1}(\Omega) \times L^{2}(\Omega)\right)$ for all time. Recall that for Systems (3) and (5), the null-controllability is equivalent to the exact controllability. As a consequence, it is not possible, taking for instance zero as initial data to reach any target state in $\left(H_{0}^{1}(\Omega)\right)^{2} \times\left(L^{2}(\Omega)\right)^{2}$ (resp. $\left.\left(L^{2}(\Omega)\right)^{2} \times\left(H^{-1}(\Omega)\right)^{2}\right)$. The controllability question for (3)-(5) hence becomes: Starting from rest at time $t=0$, is it possible to reach all $H_{0}^{1}(\Omega) \times H^{2} \cap H_{0}^{1}(\Omega) \times L^{2}(\Omega) \times H_{0}^{1}(\Omega)\left(\right.$ resp. $\left.L^{2}(\Omega) \times H_{0}^{1}(\Omega) \times H^{-1}(\Omega) \times L^{2}(\Omega)\right)$ in time $t=T$ sufficiently large? 
The strategy we adopt here is to prove some controllability results for the hyperbolic systems (3) and (5), extending the two-levels energy method introduced in [2]. Then, using transmutation techniques, we deduce controllability properties of (2) and (4).

To state our results, we recall the classical Geometric Control Conditions GCC (resp. GCC $_{\partial}$ ), which, according to [5], is a necessary and sufficient condition for the internal (resp. boundary) observability and controllability of one wave equation. We say that $\omega \subset \Omega$ satisfies GCC (resp. $\Gamma \subset \partial \Omega$ satisfies $\mathrm{GCC}_{\partial}$ ) if every generalized geodesic traveling at speed one in $\Omega$ meets $\omega$ (resp. meets $\Gamma$ on a non-diffractive point) in finite time.

We shall make the following key assumptions:

(i) We have $\left(\left(-\Delta_{c}+a\right) u, u\right)_{L^{2}(\Omega)} \geq \lambda_{0}\|u\|_{L^{2}(\Omega)}^{2}$, for some $\lambda_{0}>0$, for all $u \in L^{2}(\Omega)$. In the case where $c=\operatorname{Id}$ and $a=0$, the best constant $\lambda_{0}$ is $1 / C_{\mathcal{P}}^{2}$, where $C_{\mathcal{P}}$ is the Poincaré's constant of $\Omega$.

(ii) We have $p \geq 0$ on $\Omega,\{p>0\} \supset \bar{\omega}_{p}$ for some open subset $\omega_{p} \subset \Omega$ and set $p^{+}:=\|p\|_{L^{\infty}(\Omega)}$,

(iii) We have $b \geq 0$ on $\Omega,\{b>0\} \supset \bar{\omega}_{b}$ (resp. $b_{\partial} \geq 0$ on $\partial \Omega$ and $\left\{b_{\partial}>0\right\} \supset \bar{\Gamma}_{b}$ ) for some open subset $\omega_{b} \subset \Omega$ (resp. $\left.\Gamma_{b} \subset \partial \Omega\right)$.

We shall also require that the operator $A_{\delta}$ satisfies, for $C>0,\left(A_{\delta}\left(v_{1}, v_{2}\right),\left(v_{1}, v_{2}\right)\right)_{\delta} \geq C\left(\left\|v_{1}\right\|_{H_{0}^{1}(\Omega)}^{2}+\delta\left\|v_{2}\right\|_{H_{0}^{1}(\Omega)}^{2}\right)$ for all $\left(v_{1}, v_{2}\right) \in \mathcal{D}\left(A_{\delta}\right)$. This is the case when assuming $\sqrt{\delta} p^{+}<\lambda_{0}$.

Theorem 2.1 (Wave-type systems). Suppose that $(i)$ holds, that $\omega_{p}$ satisfies GCC and that $\omega_{b}$ (resp. $\left.\Gamma_{b}\right)$ satisfies GCC (resp. $\left.G C C_{\partial}\right)$. Then, there exists a constant $\delta_{*}>0$ such that for all $\left(\delta, p^{+}\right)$satisfying $\sqrt{\delta} p^{+}<\delta_{*}$, there exists a time $T_{*}>0$ such that for all $T>T_{*}$, all $p, b$ (resp. $b_{\partial}$ ) satisfying (ii) and (iii), and all initial data $\left(u_{1}^{0}, u_{2}^{0}, u_{1}^{1}, u_{2}^{1}\right) \in H_{0}^{1}(\Omega) \times H^{2} \cap H_{0}^{1}(\Omega) \times$ $L^{2}(\Omega) \times H_{0}^{1}(\Omega)\left(\right.$ resp. $\left.\left(u_{1}^{0}, u_{2}^{0}, u_{1}^{1}, u_{2}^{1}\right) \in L^{2}(\Omega) \times H_{0}^{1}(\Omega) \times H^{-1}(\Omega) \times L^{2}(\Omega)\right)$, there exists a control $f \in L^{2}((0, T) \times \Omega)($ resp. $f \in L^{2}((0, T) \times \partial \Omega)$ ) such that the solution of (3) (resp. (5)) satisfies $\left.\left(u_{1}, u_{2}, u_{1}^{\prime}, u_{2}^{\prime}\right)\right|_{t=T}=0$.

Another way to formulate this result is to say that, under the assumptions of Theorem 2.1, the reachable set at time $T>T_{*}$ with zero initial data is exactly $H_{0}^{1}(\Omega) \times H^{2} \cap H_{0}^{1}(\Omega) \times L^{2}(\Omega) \times H_{0}^{1}(\Omega)$ in the case of $L^{2}$ internal control and $L^{2}(\Omega) \times H_{0}^{1}(\Omega) \times H^{-1}(\Omega) \times L^{2}(\Omega)$ in the case of $L^{2}$ boundary control.

Some comments should be made about this result. First this is a generalization of the work [2] where the coupling coefficients considered have to satisfy $p(x) \geq C>0$ for all $x \in \Omega$. The geometric situations covered by Theorem 2.1 are richer, and include in particular several examples of coupling and control regions that do not intersect. Second, the coercivity assumption $(i)$ for $-\Delta_{c}+a$ together with the smallness assumption on $\sqrt{\delta} p^{+}$seem to be only technical and inherent to the method we use here. Note by the way that this smallness assumption contains the coercivity assumption for $A_{\delta}$, and allows to consider large $p^{+}$or large $\delta$ (provided that the other is small enough). Moreover, the control time $T_{*}$ we obtain depends on all the parameters of the system, and not only the sets $\omega_{p}, \omega_{b}$ and $\Gamma_{b}$ (as it is the case for a single wave equation). Finally, the fact that we consider twice the same elliptic operator $\Delta_{c}$ is a key point in our proof and it is likely that this result does not hold for waves with different speeds (see [2] for results with different speeds and different operators).

As a consequence of Theorem 2.1 and using transmutation techniques (due to [15, 11] for heat-type equations and to $[13,12]$ for Schrödinger-type equations), we can now state the associated results for Systems (2) and (4).

Corollary 2.2 (Heat-type systems). Suppose that (i) holds, that $\omega_{p}$ satisfies GCC and that $\omega_{b}$ (resp. $\left.\Gamma_{b}\right)$ satisfies GCC (resp. $\left.G C C_{\partial}\right)$. Then, there exists a constant $\delta_{*}>0$ such that for all $\left(\delta, p^{+}\right)$satisfying $\sqrt{\delta} p^{+}<\delta_{*}$, for all $T>0, \theta \in(-\pi / 2, \pi / 2)$, for all p, $b$ (resp. $\left.b_{\partial}\right)$ satisfying (ii) and (iii), and all initial data $\left(u_{1}^{0}, u_{2}^{0}\right) \in\left(L^{2}(\Omega)\right)^{2}\left(\right.$ resp. $\left.\left(u_{1}^{0}, u_{2}^{0}\right) \in\left(H^{-1}(\Omega)\right)^{2}\right)$, there exists a control $f \in L^{2}((0, T) \times \Omega)$ (resp. $\left.f \in L^{2}((0, T) \times \partial \Omega)\right)$ such that the solution of (2) (resp. (4)) satisfies $\left.\left(u_{1}, u_{2}\right)\right|_{t=T}=0$.

Corollary 2.3 (Schrödinger-type systems). Under the assumptions of Corollary 2.2, the same null-controllability result holds for System (2) (resp. (4)) with $\theta= \pm \pi / 2$, taking initial data $\left(u_{1}^{0}, u_{2}^{0}\right) \in L^{2}(\Omega) \times H_{0}^{1}(\Omega)\left(\operatorname{resp} .\left(u_{1}^{0}, u_{2}^{0}\right) \in H^{-1}(\Omega) \times L^{2}(\Omega)\right)$.

Corollary 2.2 is a direct consequence of Theorem 2.1, combined with [12, Theorem 3.4] and the smoothing effect of the heat equation. Corollary 2.3 is a direct consequence of Theorem 2.1, combined with [11, Theorem 3.1]. Since there is no smoothing effect in this case, we still obtain a controllability result in asymmetric spaces here: the uncontrolled variable $u_{2}$ has to be more regular than the other one. This shows that the attainable set from zero for a $L^{2}$ internal control (resp. $L^{2}$ boundary control) contains $L^{2}(\Omega) \times H_{0}^{1}(\Omega)\left(\right.$ resp. $\left.H^{-1}(\Omega) \times L^{2}(\Omega)\right)$. Whether or not a general target in $\left(L^{2}(\Omega)\right)^{2}($ resp. $\left.\left(H^{-1}(\Omega)\right)^{2}\right)$ is reachable for (2) (resp. (4)) with $\theta= \pm \pi / 2$ remains open.

\section{Abstract setting and ingredients of proof}

In this section, we describe the abstract setting (already used in [3]) in which we prove Theorem 2.1 for Systems (3)-(5), and define the appropriate spaces and operators. Let $H$ be a Hilbert space and $(A, \mathcal{D}(A))$ a selfadjoint positive operator on 
$H$ with compact resolvent. We denote by $(\cdot, \cdot)_{H}$ the inner product on $H$ and $\|\cdot\|_{H}$ the associated norm. For $k \in \mathbb{N}$, we set $H_{k}=\mathcal{D}\left(A^{\frac{k}{2}}\right)$ endowed with the inner product $(\cdot, \cdot)_{H_{k}}=\left(A^{\frac{k}{2}} \cdot A^{\frac{k}{2}} \cdot\right)_{H}$ and associated norm $\|\cdot\|_{H_{k}}=\left\|A^{\frac{k}{2}} \cdot\right\|_{H}$. We define $H_{-k}$ as the dual space of $H_{k}$ with respect to the pivot space $H=H_{0}$, and $\|\cdot\|_{H_{-k}}=\left\|A^{-\frac{k}{2}} \cdot\right\|_{H}$ is the norm on $H_{-k}$. The operator $A$ can be extended to an isomorphism from $H_{k}$ to $H_{k-2}$ for any $k \leq 1$, still denoted by $A$. We denote by $\lambda_{0}>0$ the largest constant satisfying $\|v\|_{H_{1}}^{2} \geq \lambda_{0}\|v\|_{H}^{2}$ for all $v \in H_{1}$, that is, the smallest eigenvalue of the selfadjoint positive operator $A$. We consider that the coupling operator $P$ is bounded on $H$ and denote by $P^{*}$ is its adjoint, $p^{+}:=\|P\|_{\mathcal{L}(H)}=\left\|P^{*}\right\|_{\mathcal{L}(H)}$. In the following, as in [2], we shall make use of the different energy levels $e_{k}(\varphi(t))=\frac{1}{2}\left(\|\varphi(t)\|_{H_{k}}^{2}+\left\|\varphi^{\prime}(t)\right\|_{H_{k-1}}^{2}\right), k \in \mathbb{Z}$ which are all preserved through time if $\varphi$ is a solution of $\varphi^{\prime \prime}+A \varphi=0$.

Before addressing the control problem, let us introduce the adjoint system

$$
\left\{\begin{array}{l}
v_{1}^{\prime \prime}+A v_{1}+\delta P v_{2}=0 \\
v_{2}^{\prime \prime}+A v_{2}+P^{*} v_{1}=0 \\
\left.\left(v_{1}, v_{2}, v_{1}^{\prime}, v_{2}^{\prime}\right)\right|_{t=0}=\left(v_{1}^{0}, v_{2}^{0}, v_{1}^{1}, v_{2}^{1}\right)
\end{array}\right.
$$

which shall stand for our observation system. This system can be recast as a first order differential equation $\mathcal{V}^{\prime}=\mathcal{A}_{\delta} \mathcal{V}$, $\mathcal{V}(0)=\mathcal{V}^{0}$, where

$$
\mathcal{A}_{\delta}=\left(\begin{array}{cc}
0 & \mathrm{Id} \\
-A_{\delta} & 0
\end{array}\right), \quad A_{\delta}=\left(\begin{array}{cc}
A & \delta P \\
P^{*} & A
\end{array}\right), \quad V=\left(v_{1}, v_{2}\right), \quad \mathcal{V}=\left(V, V^{\prime}\right)=\left(v_{1}, v_{2}, v_{1}^{\prime}, v_{2}^{\prime}\right) .
$$

Note that the operator $A_{\delta}$ is selfadjoint on the space $H \times H$ endowed with the weighted inner product $(V, \tilde{V})_{\delta}=\left(v_{1}, \tilde{v}_{1}\right)_{H}+$ $\delta\left(v_{2}, \tilde{v}_{2}\right)_{H}$. Since we have $\left(A_{\delta} V, V\right)_{\delta}=\left(A v_{1}, v_{1}\right)_{H}+\delta\left(A v_{2}, v_{2}\right)_{H}+2 \delta\left(P v_{2}, v_{1}\right)_{H} \geq\left(1-\frac{p^{+} \sqrt{\delta}}{\lambda_{0}}\right)\left(\left\|v_{1}\right\|_{H_{1}}^{2}+\delta\left\|v_{2}\right\|_{H_{1}}^{2}\right)$, we shall suppose that $p^{+} \sqrt{\delta}<\lambda_{0}$, so that $A_{\delta}$ is coercive. Under this assumption, $\left(A_{\delta}^{\frac{1}{2}} V, A_{\delta}^{\frac{1}{2}} \tilde{V}\right)_{\delta}$ defines an inner product on $\left(H_{1}\right)^{2}$, equivalent to the natural one. Assuming that $P, P^{*} \in \mathcal{L}\left(H_{k}\right)$ and writing $\mathcal{H}_{k}=\left(H_{k}\right)^{2} \times H_{k-1}^{2}, k \in \mathbb{Z}$, the operator $\mathcal{A}_{\delta}$ is an isomorphism from $\mathcal{H}_{k}$ to $\mathcal{H}_{k-1}$ and is skewadjoint on $\mathcal{H}_{k}$, equipped with the inner product $((U, V),(\tilde{U}, \tilde{V}))_{\mathcal{H}_{k}}=\left(A_{\delta}^{\frac{k}{2}} U, A_{\delta}^{\frac{k}{2}} \tilde{U}\right)_{\delta}+\left(A_{\delta}^{\frac{k-1}{2}} V, A_{\delta}^{\frac{k-1}{2}} \tilde{V}\right)_{\delta}$. Note that this is an inner product according to the coercivity assumption for $A_{\delta}$, which is equivalent to the natural inner product of $\mathcal{H}_{k}$. Hence, $\mathcal{A}_{\delta}$ generates a group $e^{t \mathcal{H}_{\delta}}$ on $\mathcal{H}_{k}$, and the homogeneous problem (6) is well-posed in these spaces. An important feature of solutions $\mathcal{V}(t)$ of System (6) is that all energies $E_{k}(\mathcal{V}(t))=1 / 2\|\mathcal{V}(t)\|_{\mathcal{H}_{k}}^{2}$ are positive and preserved through time.

For this system, now studied in $\mathcal{H}_{1}$, we shall observe only the state of the first component, i.e. $\left(u_{1}, u_{1}^{\prime}\right)$, and hence define an observation operator $\mathcal{B}^{*} \in \mathcal{L}\left(H_{2} \times H, Y\right)$, where $Y$ is a Hilbert space, standing for our observation space. This definition is sufficiently general to take into account both the boundary observation problem (taking $\mathcal{B}^{*} \in \mathcal{L}\left(H_{2}, Y\right)$ ) and the internal observation problem (taking $\mathcal{B}^{*} \in \mathcal{L}(H, Y)$ ). We assume that $\mathcal{B}^{*}$ is an admissible observation for one equation:

$$
\left\{\begin{array}{c}
\text { For all } T>0 \text { there exists a constant } C>0, \text { such that all the solutions } \varphi \text { of } \varphi^{\prime \prime}+A \varphi=f \text { satisfy } \\
\qquad \int_{0}^{T}\left\|\mathcal{B}^{*}\left(\varphi, \varphi^{\prime}\right)\right\|_{Y}^{2} d t \leq C\left(e_{1}(\varphi(0))+e_{1}(\varphi(T))+\int_{0}^{T} e_{1}(\varphi(t)) d t+\int_{0}^{T}\|f\|_{H}^{2} d t\right) .
\end{array}\right.
$$

Under this assumption, we have the following lemma.

Lemma 3.1. The operator $\mathcal{B}^{*}$ is an admissible observation for (6). More precisely, for all $T>0$, there exists a constant $C>0$, such that all the solutions of (6) satisfy

$$
\int_{0}^{T}\left\|\mathcal{B}^{*}\left(v_{1}, v_{1}^{\prime}\right)(t)\right\|_{Y}^{2} d t \leq C\left\{e_{1}\left(v_{1}(0)\right)+e_{0}\left(v_{2}(0)\right)\right\}
$$

Note that only the $e_{0}$ energy level of the second component $v_{2}$ is necessary in this admissibility estimate. Hence, we cannot hope to observe the whole $\mathcal{H}_{1}$ energy of $\mathcal{V}$ and the best observability we can expect only involves $e_{0}\left(v_{2}\right)$. Our aim is now to prove this inverse inequality of (7). For this, we have to suppose some additional assumptions on the operators $P$ and $\mathcal{B}^{*}$. Let us first precise Assumption (A2), related with the operator $P$ :

$$
\left\{\begin{array}{c}
\text { We have }\|P v\|_{H}^{2} \leq p^{+}(P v, v)_{H} \text { and there exists an operator } \Pi_{P} \in \mathcal{L}(H),\left\|\Pi_{P}\right\|_{\mathcal{L}(H)}=1, \\
\text { and a number } p^{-}>0 \text { such that }(P v, v)_{H} \geq p^{-}\left\|\Pi_{P} v\right\|_{H}^{2} \quad \forall v \in H .
\end{array}\right.
$$

Note that $p^{-} \leq p^{+}=\|P\|_{\mathcal{L}(H)}$ and that (A2) implies that the operators $P$ and $P^{*}$ are non-negative. In the applications to coupled wave equations, $P$ is the multiplication by the function $p$ and the operator $\Pi_{P}$ is the multiplication by the characteristic function $\mathbb{1}_{\omega_{p}}$. Next, we shall suppose that a single equation is observable both by $\mathcal{B}^{*}$ and by $\Pi_{P}$ in sufficiently large time:

$$
\left\{\begin{array}{c}
\exists T_{0}>0 \text { such that for all } T>T_{0} \text { there exists a constant } C>0, \text { such that all solutions } \varphi \text { of } \varphi^{\prime \prime}+A \varphi=0 \\
\text { satisfy both } e_{1}(\varphi(0)) \leq C \int_{0}^{T}\left\|\mathcal{B}^{*}\left(\varphi, \varphi^{\prime}\right)\right\|_{Y}^{2} \text { and } \quad e_{1}(\varphi(0)) \leq C \int_{0}^{T}\left\|\Pi_{P} \varphi^{\prime}\right\|_{H}^{2} d t
\end{array}\right.
$$


In the context of Theorem 2.1, these observability assumptions are satisfied as soon as $\omega_{p}$ and $\omega_{b}$ satisfy GCC (resp. $\Gamma_{b}$ satisfies $\mathrm{GCC}_{\partial}$ ). We can now state (without proof) the main result of this note.

Theorem 3.2. Suppose that Assumptions (A1)-(A3) hold. Then there exists a constant $\delta_{*}$ such that for all $\left(\delta, p^{+}\right)$satisfying $\sqrt{\delta} p^{+}<\delta_{*}$, there exists a time $T_{*}$ such that for all $T>T_{*}$ there exists $C>0$, such that for all $\mathcal{V}^{0} \in \mathcal{H}_{1}$, the solution $\mathcal{V}(t)=e^{t \mathcal{A}_{\delta}} \mathcal{V}^{0}$ of $(6)$ satisfies

$$
e_{1}\left(v_{1}(0)\right)+e_{0}\left(v_{2}(0)\right) \leq C \int_{0}^{T}\left\|\mathcal{B}^{*}\left(v_{1}, v_{1}^{\prime}\right)(t)\right\|_{Y}^{2} d t
$$

Applying the Hilbert Uniqueness Method (HUM) of [10], we deduce now controllability results for the adjoint system. In this context, we have to define more precisely the observation operator. We shall treat two cases: First, $\mathcal{B}^{*}\left(v_{1}, v_{1}^{\prime}\right)=B^{*} v_{1}^{\prime}$ with $B^{*} \in \mathcal{L}(H, Y)$, corresponding to internal observability (with $Y=L^{2}(\Omega)$ ), and second $\mathcal{B}^{*}\left(v_{1}, v_{1}^{\prime}\right)=B^{*} v_{1}$ with $B^{*} \in$ $\mathcal{L}\left(H_{2}, Y\right)$, corresponding to boundary observability (with $Y=L^{2}(\partial \Omega)$ ). In both cases, we define the control operator $B$ as the adjoint of $B^{*}$, and the control problem reads, for a control function $f$ taking its values in $Y$,

$$
\left\{\begin{array}{l}
u_{1}^{\prime \prime}+A u_{1}+\delta P u_{2}=B f \\
u_{2}^{\prime \prime}+A u_{2}+P^{*} u_{1}=0 \\
\left.\left(u_{1}, u_{2}, u_{1}^{\prime}, u_{2}^{\prime}\right)\right|_{t=0}=\left(u_{1}^{0}, u_{2}^{0}, u_{1}^{1}, u_{2}^{1}\right) .
\end{array}\right.
$$

This is an abstract version of (3)-(5). Note that under this form, System (9) not only contains (3)-(5), but also locally coupled systems of plate equations, with a distributed or a boundary control.

First case: $\mathcal{B}^{*}\left(v_{1}, v_{1}^{\prime}\right)=B^{*} v_{1}^{\prime}$ with $B^{*} \in \mathcal{L}(H, Y)$. In this case, $B \in \mathcal{L}(Y, H)$ and the control problem (9) is well-posed in $\mathcal{H}_{1}$ for $f \in L^{2}(0, T ; Y)$. In this setting, we first deduce from (8) the following other observability estimate for solutions $\mathcal{W}$ of (6) in $\mathcal{H}_{0}: e_{0}\left(w_{1}(0)\right)+e_{-1}\left(w_{2}(0)\right) \leq C \int_{0}^{T}\left\|B^{*} w_{1}(t)\right\|_{Y}^{2} d t$. The internal control result of Theorem 2.1 is then a direct consequence of the HUM since Assumptions (A1)-(A3) are satisfied in this application.

Second case: $\mathcal{B}^{*}\left(v_{1}, v_{1}^{\prime}\right)=B^{*} v_{1}$ with $B^{*} \in \mathcal{L}\left(H_{2}, Y\right)$. As a consequence of the admissibility inequality (7), System (9) is well-posed in $\mathcal{H}_{0}$ in the sense of transposition solutions. In this setting, the boundary control result of Theorem 2.1 is a direct consequence of the HUM and Theorem 3.2 since Assumptions (A1)-(A3) are satisfied in this application.

\section{References}

[1] F. Alabau-Boussouira. Indirect boundary stabilization of weakly coupled systems. SIAM J. Control Optim., 41:511-541, 2002.

[2] F. Alabau-Boussouira. A two-level energy method for indirect boundary observability and controllability of weakly coupled hyperbolic systems. SIAM J. Control Optim., 42:871-906, 2003.

[3] F. Alabau-Boussouira and M. Léautaud. Indirect stabilization of locally coupled wave-type systems. to appear in ESAIM Control Optim. Calc. Var., 2011.

[4] F. Ammar-Khodja, A. Benabdallah, and C. Dupaix. Null controllability of some reaction-diffusion systems with one control force. J. Math. Anal. Appl., 320:928-943, 2006.

[5] C. Bardos, G. Lebeau, and J. Rauch. Sharp sufficient conditions for the observation, control, and stabilization of waves from the boundary. SIAM J. Control Optim., 30:1024-1065, 1992.

[6] E. Fernández-Cara, M. González-Burgos, and L. de Teresa. Boundary controllability of parabolic coupled equations. J. Funct. Anal., 259(7):1720$1758,2010$.

[7] M. González-Burgos and R. Pérez-García. Controllability results for some nonlinear coupled parabolic systems by one control force. Asymptotic Analysis, 46:123-162, 2006.

[8] O. Kavian and L. de Teresa. Unique continuation principle for systems of parabolic equations. ESAIM Control Optim. Calc. Var., 16(2):247-274, 2010.

[9] M. Léautaud. Spectral inequalities for non-selfadjoint elliptic operators and application to the null-controllability of parabolic systems. J. Funct. Anal., 258:2739-2778, 2010.

[10] J.-L. Lions. Contrôlabilité exacte, perturbations et stabilisation de systèmes distribués. Tome 1, volume 8 of Recherches en Mathématiques Appliquées [Research in Applied Mathematics]. Masson, Paris, 1988.

[11] L. Miller. Controllability cost of conservative systems: resolvent condition and transmutation. J. Funct. Anal., 218(2):425-444, 2005.

[12] L. Miller. The control transmutation method and the cost of fast controls. SIAM J. Control Optim., 45(2):762-772, 2006.

[13] K.-D. Phung. Observability and control of Schrödinger equations. SIAM J. Control Optim., 40(1):211-230, 2001.

[14] L. Rosier and L. de Teresa. Exact controllability of a cascade system of conservative equations. C. R. Acad. Sci. Paris, Ser. I, doi:10.1016/j.crma.2011.01.014, 2011.

[15] D.L. Russell. A unified boundary controllability theory for hyperbolic and parabolic partial differential equations. Studies in Appl. Math., 52:189221, 1973.

[16] L. de Teresa. Insensitizing controls for a semilinear heat equation. Comm. Partial Differential Equations, 25:39-72, 2000. 